\title{
The influence of bearing surfaces on revisions due to dislocations in total hip arthroplasty
}

\author{
Francesco Castagnini $\mathbb{D}^{1} \cdot$ Barbara Bordini $^{2} \cdot$ Monica Cosentino ${ }^{2} \cdot$ Cristina Ancarani $^{2} \cdot$ Federica Mariotti $^{1}$. \\ Federico Biondi ${ }^{1} \cdot$ Cesare Faldini ${ }^{3,4} \cdot$ Francesco Traina $^{4,5}$
}

Received: 4 December 2020 / Accepted: 29 August 2021 / Published online: 15 September 2021

(c) The Author(s) 2021

\begin{abstract}
Introduction Recurrent dislocations are still the most frequent reason for revision in total hip arthroplasty (THA). The impact of bearing surfaces on dislocations is still controversial. We hypothesized that: (1) bearing surfaces influence the revisions due to dislocations; (2) ceramic-on-ceramic reduced the revisions for dislocations in adjusted models; (3) Delta-onDelta bearings reduced the revisions for dislocations in comparison to surfaces with cross-linked polyethylene.

Materials and methods The regional arthroplasty registry was enquired about bearing surfaces and revisions for dislocations and instability. Unadjusted and adjusted rates were provided, including sex, age ( $<65$ years or $\geq 65$ years), head diameter ( $\leq 28 \mathrm{~mm}$ or $>28 \mathrm{~mm} ;<36 \mathrm{~mm}$ or $\geq 36 \mathrm{~mm}$ ) as variables. 44,065 THAs were included.

Results The rate of revisions for dislocations was significantly lower in ceramic-on-ceramic and metal-on-metal bearings (unadjusted rates). After adjusting for age, sex, and head size (36 and $28 \mathrm{~mm}$ ), hard-on-hard bearings were protective $(p<$ 0.05): ceramic-on-ceramic had a lower risk of revisions due to dislocation than ceramic-on-polyethylene (HR 1.6, 95\% CI $1.2-2.2 p=0.0009)$. The rate of revisions for dislocation was similar in bearings with cross-linked polyethylene and Deltaon-Delta articulations, in unadjusted and adjusted models.

Conclusion Bearings with conventional polyethylene were more predisposed to dislocations. Currently adopted bearings exerted no significant influence on revisions due to dislocations. These findings could be primarily related to wear, but due to the time distribution, soft tissue envelopes and surface tension may also play a role. Pre-clinical biomechanical evaluations and prospective matched cohort studies are required to draw definitive conclusions.
\end{abstract}

\section{Graphical Abstract}
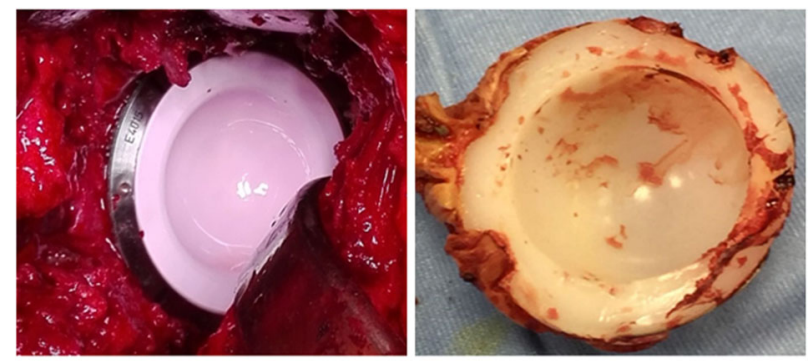

Hard-on-hard bearings have lower risk of revisions due to dislocations in

comparison to surfaces with conventional polyethylene

Revisions for dislocation are similar in bearings with cross-linked polyethylene

and Delta-on-Delta articulations

Supplementary information The online version contains supplementary material available at https://doi.org/10.1007/s10856021-06598-4.

Francesco Castagnini

francescocastagnini@hotmail.it

Extended author information available on the last page of the article

\section{Introduction}

Dislocations are still the most frequent reason for revision in total hip arthroplasty (THA) and the incidence is steadily rising $[1,2]$. Head size, patient's age, surgeon experience, surgical approach and implant type have been advocated as the major 
predictive factors influencing THA dislocations [3-5]. The role of bearing surfaces in preventing dislocations has been discussed, with controversial conclusions. While Hernigou et al. reported that ceramic couplings were less susceptible to dislocations, Sexton et al. noted that the revision rates due to dislocations were statistically higher in primary ceramic-onceramic THAs $[3,6,7]$. The two most recent registry studies by Pitto et al. and Shah et al. concluded that bearing surfaces have a little, non-significant impact on revisions for dislocations $[2,8]$.

Thus, the current literature about bearing surfaces and revisions for dislocations is contradictive and only partially examines the most recent bearing surfaces, Delta ceramics and cross-linked polyethylene (XLPE) [8]. Hence, a large registry population of primary THAs was investigated to assess whether: (1) bearing surfaces impacted the revision rates due to dislocations (unadjusted rates); (2) ceramic-onceramic reduced the revisions for dislocations after model adjustment for gender, age ( $<65$ years or $\geq 65$ years), head diameter ( $\leq 28$ or $>28 \mathrm{~mm}$; $<36$ or $\geq 36 \mathrm{~mm}$ ); (3) Delta-onDelta bearings reduced the revision rates due to dislocations in comparison to modern articulations as metal-on-XLPE and Delta-on-XLPE in unadjusted and adjusted populations.

\section{Materials and methods}

The Emilia Romagna region registry RIPO includes primary and revision hip, knee and shoulder arthroplasties, performed in 68 Orthopedic Units since January 2000 [5, 9]. The demographics of patients, the diagnoses leading to THA, the features of surgical procedures, the type (batch and code) and fixation of implants are collected similarly to the most important national registries. A specific paper form including all the data is filled in by the surgeon and then it is sent to RIPO. Crossover comparisons with other databases and missing data retrievals are routinely performed to improve registry accuracy. The report includes only resident patients. This method avoids the bias due to the lack of follow-up data: surgical revisions on resident patients, even when performed elsewhere in Italy, are always billed back to the reference region and captured by the registry. RIPO achieved a capture rate of $98 \%[5,9]$.

RIPO was enquired about the correlation between bearing surfaces and rates of revisions for primary instability/dislocations in primary cementless THAs. The inclusion criteria of the present study were all the primary cementless THAs performed for primary osteoarthritis and avascular necrosis, from January 2000 to December 2016.

The exclusion criteria were:

-THAs performed for diagnoses other than primary osteoarthritis or avascular necrosis -cemented or hybrid implants -dual mobility articulations

-hip resurfacing procedures

-metal-on-metal THAs with heads larger than $36 \mathrm{~mm}$

The exclusion criteria aimed to exclude patients with higher risks of dislocation (e.g., dysplastic) and all the implants adopted in high-risk patients (e.g., dual mobility) or correlated with soft tissue pathology (metal-on-metal implants with large heads possibly causing metallosis) $[3,10]$.

Demographics and implant features were collected and compared. The end-points of the study were revisions for dislocations (multiple dislocation occurring after three months) and revisions for primary instability (revisions due to subluxation/impingement related events occurring in the first three months) [5].

First, an unadjusted evaluation of bearings and revisions due to dislocations was performed. Then, bearings were compared using a regression model to assess the effects of independent predictive factors for dislocations (inferred from literature) on revisions due to dislocations/primary instability: sex, age, head size [2, 3]. Lastly, the most widespread and recent articulations, Delta-on-Delta (Biolox Delta, Ceramtec, Plochingen, Germany), Delta-on-XLPE, metal-on-XLPE, were compared. Oxinium-on-XLPE (Smith and Nephew, Andover, US) bearings were not evaluated due to the low number of implants involved.

44,065 THAs met the inclusion criteria and were analyzed (Table 1). Ceramic-on-ceramic (COC) surfaces were implanted in the vast majority of THAs (53\%). Ceramic-on-polyethylene (COP) articulations accounted for the $26.4 \%$ of the whole implants, whereas metal-on-metal (MOM) $(0.4 \%)$ and metalon-polyethylene (MOP) (17.1\%) were occasionally adopted. $293(0.7 \%)$ cases were revised due to recurrent dislocations or primary instability. THAs were stratified by bearing surfaces: demographics and implant features were detailed in Table 1.

Table 1 Demographics and implant features were similar in the four groups

\begin{tabular}{lllll}
\hline Demographics and implant features & & & \\
\hline & COC & COP & MOM & MOP \\
\hline Number of implants & 23,348 & 11,630 & 1560 & 7527 \\
Mean age (years) & 66.6 & 71.5 & 63.9 & 71.9 \\
Female sex (\%) & $55 \%$ & $58 \%$ & $55 \%$ & $60 \%$ \\
BMI $\left(\mathrm{kg} / \mathrm{m}^{2}\right)$ between $19-25$ & $32 \%$ & $34 \%$ & $35 \%$ & $33 \%$ \\
Weight $<80 \mathrm{~kg}(\%)$ & $63 \%$ & $67 \%$ & $65 \%$ & $66 \%$ \\
Head size $<36 \mathrm{~mm}(\%)$ & $44 \%$ & $73 \%$ & $100 \%$ & $92 \%$ \\
Head size $\leq 28 \mathrm{~mm}(\%)$ & $14 \%$ & $47 \%$ & $85 \%$ & $78 \%$ \\
Lateral approach $(\%)$ & $48 \%$ & $61 \%$ & $80 \%$ & $69 \%$ \\
Posterolateral approach $(\%)$ & $32 \%$ & $33 \%$ & $19 \%$ & $25 \%$ \\
Revisions for dislocation & 110 & 100 & 8 & 75 \\
Follow-up (years) & 5.6 & 6.1 & 10.1 & 8.4 \\
\hline
\end{tabular}


Table 2 Demographic and implant features of the most recent articulations (Delta-on-Delta, Delta-on-XLPE, metal-on-XLPE) showed inhomogeneous distribution between the three cohorts

\begin{tabular}{llll}
\hline Demographics and implant features & & \\
\hline & $\begin{array}{l}\text { Delta-on- } \\
\text { Delta }\end{array}$ & $\begin{array}{l}\text { Delta-on- } \\
\text { XLPE }\end{array}$ & $\begin{array}{l}\text { Metal-on- } \\
\text { XLPE }\end{array}$ \\
\hline Number of implants & 16,672 & 4648 & 3179 \\
Mean age (years) & 67 & 72.6 & 73 \\
Female sex (\%) & $54 \%$ & $56 \%$ & $60 \%$ \\
BMI $\left(\mathrm{kg} / \mathrm{m}^{2}\right)$ between & $32 \%$ & $33 \%$ & $34 \%$ \\
19-25 & & & \\
Weight $<80 \mathrm{~kg}(\%)$ & $63 \%$ & $63 \%$ & $66 \%$ \\
Head size $<36 \mathrm{~mm}(\%)$ & $32 \%$ & $51 \%$ & $81 \%$ \\
Head size $\leq 28 \mathrm{~mm}(\%)$ & $1 \%$ & $13 \%$ & $66 \%$ \\
Lateral approach (\%) & $44 \%$ & $55 \%$ & $71 \%$ \\
Posterolateral & $33 \%$ & $36 \%$ & $21 \%$ \\
approach $(\%)$ & & & \\
Revisions for dislocation & 66 & 27 & 21 \\
Follow-up (years) & 3.9 & 3 & 7 \\
\hline
\end{tabular}

Similarly, the demographics and implant features of the most recent articulations were specified in Table 2 .

Institutional board review was waived due to the nature of registry studies, collecting anonymous data as a standard practice.

\section{Statistical analysis}

Patient demographics, implant features and reasons for revision were analyzed using descriptive statistics, such as means, ranges, and percentages. Values were compared using a $t$-test or Chi-square test. Kaplan-Meier survival analysis was performed. Survival times of unrevised implants were calculated considering the last date of observation or the date of death. The log-rank test was used to compare survivorships of the cohorts. Survival data were analyzed using Cox multiple regression model: Wald test was used to calculate the $p$ values. The proportionality hazards assumption (HR) was tested by the Schoenfeld residual method; age, gender and head sizes used for adjustment fulfilled the proportional hazard assumption for the whole period. The threshold for significance was $p=0.05$ for all the tests. All statistical analyses were performed using $\mathrm{JMP}^{\circledast}$, Version $<\mathrm{x}>$. SAS Institute Inc., Cary, NC, 1989-2007.

\section{Results}

The risk of revision for dislocations was significantly lower for hard-on-hard bearings (COC and MOM) than for

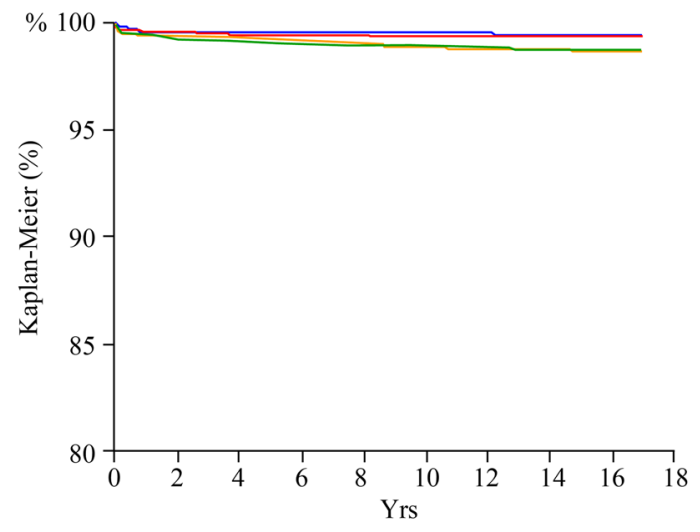

\begin{tabular}{|c|c|c|c|c|c|c|}
\hline & \multicolumn{6}{|c|}{$\%$ Survival rate (CI 95\%) } \\
\hline & $1 \mathrm{Yr}$ & $3 \mathbf{Y r}$ & $5 \mathrm{Yr}$ & $7 \mathrm{Yr}$ & $10 \mathrm{Yr}$ & $15 \mathrm{Yr}$ \\
\hline \multirow[t]{2}{*}{$\mathrm{COC}$} & 99.6 & 99.6 & 99.5 & 99.5 & 99.4 & 99.4 \\
\hline & (99.6-99.7) & $(99.5-99.7)$ & (99.4-99.6) & (99.4-99.6) & $(99.3-99.5)$ & $(99.2-99.5)$ \\
\hline Prostheses at risk & 20893 & 16037 & 11544 & 7508 & 3313 & 426 \\
\hline \multirow[t]{2}{*}{ COP } & 99.5 & 99.3 & 99.1 & 99.0 & 98.9 & 98.8 \\
\hline & (99.3-99.6) & $(99.1-99.4)$ & (98.9-99.3) & (98.8-99.2) & $(98.6-99.1)$ & $(98.4-99.0)$ \\
\hline Prostheses at risk & 10053 & 7659 & 5904 & 4446 & 2738 & 530 \\
\hline \multirow[t]{2}{*}{ MOM } & 99.5 & 99.5 & 99.5 & 99.5 & 99.5 & 99.4 \\
\hline & (99.1-99.8) & (99.1-99.8) & (99.1-99.8) & $(99.1-99.8)$ & $(99.1-99.8)$ & $(98.6-99.7)$ \\
\hline Prostheses at risk & 1532 & 1468 & 1373 & 1221 & 832 & 176 \\
\hline \multirow[t]{2}{*}{ MOP } & 99.4 & 99.3 & 99.2 & 99.1 & 98.9 & 98.6 \\
\hline & $(99.2-99.6)$ & (99.1-99.5) & $(99.0-99.4)$ & $(98.8-99.3)$ & $(99.6-99.1)$ & $(98.1-99.0)$ \\
\hline Prostheses at risk & 7122 & 6386 & 5577 & 4661 & 2898 & 549 \\
\hline
\end{tabular}

Fig. 1 The Kaplan-Meier curves (endpoint: revisions due to recurrent dislocations and primary instability) showed that COC implants were significantly less prone to dislocations than COP THAs (unadjusted rates). COC: red line; COP: green line; MOM: blue line; MOP: orange line

hard-on-soft articulations (COP and MOP) (unadjusted rates; $p<0.05$ ) (Fig. 1).

The adjusted risk ratios for gender, age $(<65$ years or $\geq 65$ years), head diameter $(\leq 28 \mathrm{~mm}$ or $>28 \mathrm{~mm} ;<36 \mathrm{~mm}$ or $\geq 36 \mathrm{~mm}$ ) were calculated using the Cox multiple regression model.

The regression model adjusted for age gender and head diameter $(28 \mathrm{~mm})$ showed that bearing surfaces statistically influenced the revisions due to dislocations $(p=0.03)$ (Table 3). COP couplings were more at risk of dislocations than COC (HR 1.4, 95\% CI 1.0-1.9, $p=0.04$ ) and MOM (HR $2.4,95 \%$ CI 1.2-5.4, $p=0.008$ ). MOP articulations were more prone to dislocations than MOM (HR 2.1, 95\% CI 1.1-4.8, $p=0.03)$. No differences were detected between MOM and COC couplings (HR 0.6, 95\% CI 0.3-1.1, $p=0.1$ ).

When the model was adjusted for age, gender, and head diameter $(36 \mathrm{~mm})$, once again bearing surfaces influenced the rate of revisions for dislocations $(p=0.001)$ (Table 4). There was a higher risk of revisions due to dislocations in COP cohort than in COC implants (HR 1.6, 95\% CI 1.2-2.2 $p=0.0009$ ) and in MOM THAs (HR 2.2, 95\% CI 1.1-4.9, $p=0.02)$. No differences were detected between MOM and COC articulations (HR 0.7, 95\% CI 0.3-1.4, $p=0.4$ ).

When the most recent articulations were analyzed (metalon-XLPE, Delta-on-XLPE, Delta-on-Delta), the survival 
Table 3 Cox regression model investigating the influence of bearings on revisions for dislocation was adjusted for age, gender, and head diameter $(28 \mathrm{~mm})$ : in bold, significant findings

\begin{tabular}{lllll}
\hline Bearings & & HR & CI 95\% & $p$ \\
\hline COC & MOM & 1.74 & $0.88-3.95$ & 0.1148 \\
COC & COP & $\mathbf{0 . 7 3}$ & $\mathbf{0 . 5 4 - 0 . 9 8}$ & $\mathbf{0 . 0 3 8 4}$ \\
COC & MOP & 0.82 & $0.58-1.16$ & 0.2637 \\
MOM & COC & 0.57 & $0.25-1.13$ & 0.1148 \\
MOM & COP & $\mathbf{0 . 4 1}$ & $\mathbf{0 . 1 9 - 0 . 8 1}$ & $\mathbf{0 . 0 0 8 2}$ \\
MOM & MOP & $\mathbf{0 . 4 7}$ & $\mathbf{0 . 2 1 - 0 . 9 2}$ & $\mathbf{0 . 0 2 7 3}$ \\
COP & COC & $\mathbf{1 . 3 7}$ & $\mathbf{1 . 0 1 - 1 . 8 5}$ & $\mathbf{0 . 0 3 8 4}$ \\
COP & MOM & $\mathbf{2 . 3 9}$ & $\mathbf{1 . 2 3 - 5 . 3 7}$ & $\mathbf{0 . 0 0 8 2}$ \\
COP & MOP & 1.13 & $0.83-1.53$ & 0.4354 \\
MOP & COC & 1.22 & $0.86-1.71$ & 0.2637 \\
MOP & MOM & $\mathbf{2 . 1 2}$ & $\mathbf{1 . 0 8}-\mathbf{4 . 7 9}$ & $\mathbf{0 . 0 2 7 3}$ \\
MOP & COP & 0.88 & $0.65-1.20$ & 0.4354 \\
\hline
\end{tabular}

Table 4 Cox regression model investigating the influence of bearings on revisions for dislocation was adjusted for age, gender, and head diameter $(36 \mathrm{~mm})$ : in bold, significant findings

\begin{tabular}{lllll}
\hline Bearings & & HR & CI 95\% & $p$ \\
\hline COC & MOM & 1.35 & $0.69-3.03$ & 0.4070 \\
COC & COP & $\mathbf{0 . 6 1}$ & $\mathbf{0 . 4 6}-\mathbf{0 . 8 2}$ & $\mathbf{0 . 0 0 0 9}$ \\
COC & MOP & $\mathbf{0 . 6 3}$ & $\mathbf{0 . 4 6}-\mathbf{0 . 8 7}$ & $\mathbf{0 . 0 0 4 9}$ \\
MOM & COC & 0.74 & $0.33-1.45$ & 0.4070 \\
MOM & COP & $\mathbf{0 . 4 5}$ & $\mathbf{0 . 2 0 - 0 . 8 9}$ & $\mathbf{0 . 0 1 8 6}$ \\
MOM & MOP & 0.47 & $0.21-0.92$ & 0.0249 \\
COP & COC & $\mathbf{1 . 6 3}$ & $\mathbf{1 . 2 2}-\mathbf{2 . 1 6}$ & $\mathbf{0 . 0 0 0 9}$ \\
COP & MOM & $\mathbf{2 . 1 9}$ & $\mathbf{1 . 1 3 - 4 . 9 2}$ & $\mathbf{0 . 0 1 8 6}$ \\
COP & MOP & 1.02 & $0.76-1.39$ & 0.8833 \\
MOP & COC & $\mathbf{1 . 5 9}$ & $\mathbf{1 . 1 5}-\mathbf{2 . 1 9}$ & $\mathbf{0 . 0 0 4 9}$ \\
MOP & MOM & $\mathbf{2 . 1 4}$ & $\mathbf{1 . 0 9 - 4 . 8 4}$ & $\mathbf{0 . 0 2 4 9}$ \\
MOP & COP & 0.98 & $0.72-1.32$ & 0.8833 \\
\hline
\end{tabular}

rates (endpoint: revisions due to recurrent dislocations/primary instability) were similar in the three cohorts (unadjusted, $p=0.06$ ) (Fig. 2). When the three cohorts were stratified for the $28 \mathrm{~mm}$ head diameter, no significant differences between couplings were detected in terms of revisions due to dislocations $(p>0.05)$. The articular surfaces did not influence the rate of revisions for dislocations when the model was adjusted for the age, gender, and $36 \mathrm{~mm}$ head diameter $(p=0.177)$.

\section{Discussion}

Hard-on-hard (COC and MOM) bearing surfaces exerted a protective influence against revisions due to dislocations and instability when conventional polyethylene was evaluated

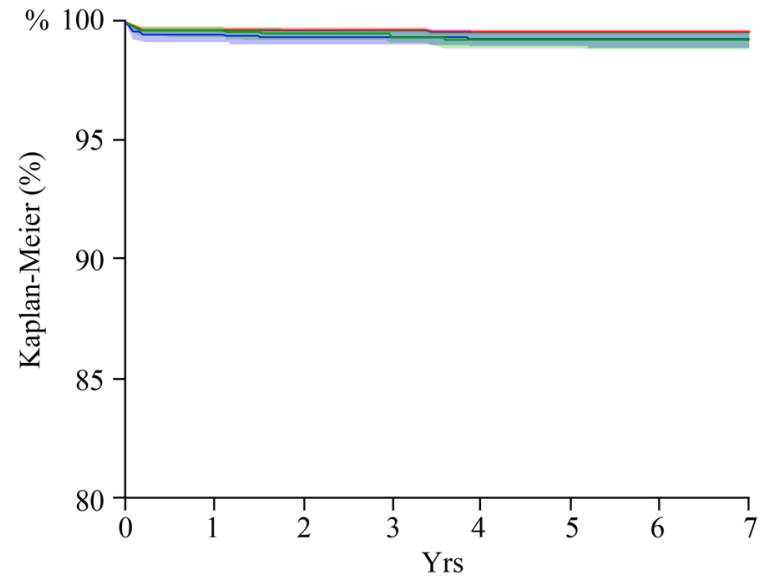

\begin{tabular}{|l|c|c|c|c|}
\cline { 2 - 5 } \multicolumn{1}{c|}{} & $\mathbf{1 Y r}$ & $\mathbf{3 Y r}$ & $\mathbf{5 Y r}$ & $\mathbf{7 Y r}$ \\
\hline Delta on Delta & 99.7 & 99.6 & 99.6 & 99.6 \\
Prostheses at risk & 14352 & 9819 & 5645 & 2155 \\
\hline Delta on XLPE & 99.6 & 99.4 & 99.2 & 99.2 \\
& $(99.3-99.7)$ & $(99.1-99.6)$ & $(98.9-99.5)$ & $(98.9-99.5)$ \\
Prostheses at risk & 3646 & 2013 & 960 & 262 \\
\hline Metal on XLPE & 99.5 & 99.4 & 99.3 & 99.3 \\
& $(99.1-99.7)$ & $(99.0-99.6)$ & $(99.0-99.6)$ & $(98.9-99.5)$ \\
Prostheses at risk & 2920 & 2420 & 1936 & 1497 \\
\hline
\end{tabular}

Fig. 2 Recent couplings did not show significant differences in terms of revision due to dislocation (Kaplan-Meier curves, endpoint: revisions due to recurrent dislocations and primary instability). Delta-onDelta: red line. Delta-on-XLPE: green line. Metal-on-XLPE: blue line

(Fig. 1). The lower rate of dislocations with hard-on-hard articular surfaces was evident even when some variables related to unstable THA (age, gender, and head size) were controlled (Tables 3 and 4). However, the recent bearings (metal-on-XLPE, Delta-on-XLPE, and Delta-on-Delta) showed no differences in terms of revisions due to dislocations and instability, in unadjusted and adjusted populations (Fig. 2).

The available literature about the impact of bearings on dislocations is controversial: many Authors found out weak correlations $[2,3,8]$. The present report seems to support the findings of Pitto et al., who noticed a weak prevalence of revisions for dislocations in the COP cohort [2]. But, at the same time, the present report was in line with Shah et al. [8]. The Authors evaluated COC and articulations with XLPE: no significant differences in terms of revisions for dislocations were evident apart from metal-on-XLPE bearings with $36 \mathrm{~mm}$ heads exhibiting more revisions [8]. The most logical explanation of the predisposition of conventional polyethylene to dislocations in comparison to hard-on-hard bearings and modern articulations with XLPE is obviously wear $[6,7,11,12]$. However, the wear of conventional polyethylene bearings may easily justify late dislocations occurring at long-terms, but in this report, conventional polyethylene liners showed higher rates of dislocation even at mid-terms (Fig. 1). Thus, advocating only for wear is arduous. 
So, other hypotheses explaining the different impact of bearings on dislocations should be made. The first hypothesis concerns the soft tissue envelopes. A thick neocapsule of clean, dense, regular fibrous tissue full of Type-1 collagen was demonstrated around COC surfaces [13, 14]. Thus, this new capsular reinforcement may effectively stabilize the hip implant even at short-to-midterms, but it was demonstrated only for COC implants [14].

A more comprehensive hypothesis concerns the surface tension [15-18]. As hard-on-hard bearings are highly wettable and smooth materials, the thin fluid film between head and liner generates restraining forces avoiding hip separation during the swing phase of the gait $[17,18]$. As a matter of fact, an in vitro evaluation by Clarke et al. demonstrated notable retaining forces for MOM implants at all speeds, more than 12 times higher than COP couplings [16]. These forces translated into the much lower dislocation rates in MOM THAs than in COP implants with the same head size [16]. Komistek et al. ascertained that MOM implants did not exhibit a femoral head separation during the swing phase, differently from MOP articulations [17]. Although literature about ceramics and the cohesiveness of lubricating film is scarce, it is very likely that COC bearings may exhibit a similar behavior, as the smoothness and the wettability of such bearings are even more pronounced than in MOM [18]. It is hard to state whether superficial tension may be advocated also for XLPE, as polymers are known for the low wettability. However, the dependable effects of XLPE articulations against dislocations were observed in other registry studies: biomechanical assessments would be helpful to explain such findings $[4,8]$.

The main limits of this report are related to the nature of registry studies [2, 3, 5]. Registries allow to detect only revision procedures: thus, conservatively treated dislocations were not captured. The multifactorial etiology of dislocation cannot be properly analyzed by a registry study $[2,3,12,13]$. Thus, some key factors as the clinical conditions of the patients, surgeon experience, impingement, component malposition and even some specific features of the implants were not assessed. In particular, a proper analysis about elevated liners was not conducted: such devices may reduce dislocation rates, even if this finding is still controversial [19]. On the other side, dislocation is quite a rare event. So, registry studies on large numbers are adequate to allow for proper comparisons, stratify groups and control independent risk factors [13].

\section{Conclusion}

Bearing surfaces with conventional polyethylene were more predisposed to revisions for dislocations and instability.
However, with modern bearings with XLPE, no influence of bearing surfaces on revisions due to dislocation is evident. While the most logical explanation of these findings would be wear, the time distribution of the dislocations advocates other causes, like soft tissue envelopes, lubricant conditions and surface tension, all possibly contributing to the better stability of the hard-on-hard and XLPE surfaces. More biomechanical studies about the fluid-film cohesion of the most recent materials and prospective matched cohort studies about the influence of articular surfaces on dislocations may implement the current knowledge about bearings and dislocations.

\section{Compliance with ethical standards}

Conflict of interest The authors declare no competing interests.

Publisher's note Springer Nature remains neutral with regard to jurisdictional claims in published maps and institutional affiliations.

Open Access This article is licensed under a Creative Commons Attribution 4.0 International License, which permits use, sharing, adaptation, distribution and reproduction in any medium or format, as long as you give appropriate credit to the original author(s) and the source, provide a link to the Creative Commons license, and indicate if changes were made. The images or other third party material in this article are included in the article's Creative Commons license, unless indicated otherwise in a credit line to the material. If material is not included in the article's Creative Commons license and your intended use is not permitted by statutory regulation or exceeds the permitted use, you will need to obtain permission directly from the copyright holder. To view a copy of this license, visit http://creativecommons. org/licenses/by/4.0/.

\section{References}

1. Wera GD, Ting NT, Moric M, Paprosky WG, Sporer SM, Della Valle CJ. Classification and management of the unstable total hip arthroplasty. J Arthroplast. 2012;27:710-5.

2. Pitto RP, Garland M, Sedel L. Are ceramic-on-ceramic bearings in total hip arthroplasty associated with reduced revision risk for late dislocation? Clin Orthop Relat Res. 2015;473:3790-5.

3. Sexton SA, Walter WL, Jackson MP, De Steiger R, Stanford T. Ceramic-on-ceramic bearing surface and risk of revision due to dislocation after primary total hip replacement. J Bone Jt Surg Br. 2009;91:1448-53.

4. Zijlstra WP, De Hartog B, Van Steenbergen LN, Scheurs BW, Nelissen RGHH. Effect of femoral head size and surgical approach on risk of revision for dislocation after total hip arthroplasty. Acta Orthop. 2017;88:395-401.

5. Giardina F, Castagnini F, Stea S, Bordini B, Montalti M, Toni A. Short stems versus conventional stems in cementless total hip arthroplasty: a long-term registry study. J Arthroplast. 2018;33:1794-9.

6. Hernigou P, Homma Y, Pidet O, Guissou I, Hernigou J. Ceramicon-ceramic bearing decreases the cumulative long-term risk of dislocation. Clin Orthop Relat Res. 2013;471:3875-82.

7. Hernigou P, Roussignol X, Delambre J, Poignard A, FlouzatLachaniette $\mathrm{CH}$. Ceramic-on-ceramic THA associated with fewer dislocations and less muscle degeneration by preserving muscle progenitors. Clin Orthop Relat Res. 2015;473:3762-9. 
8. Shah SM, Walter WL, Tai SM, Lorimer MF, de Steiger RN. Late dislocations after total hip arthroplasty: is the bearing a factor? $\mathrm{J}$ Arthroplast. 2017;32:2852-6.

9. Bordini B, Stea S, Castagnini F, Busanelli L, Giardina F, Toni A. The influence of bearing surfaces on periprosthetic hip infections: analysis of thirty nine thousand, two hundred and six cementless total hip arthroplasties. Int Orthop. 2018. https://doi.org/10.1007/ s00264-018-4097-2.

10. de Steiger R, Peng A, Lewis P, Graves S. What is the long-term survival for primary THA with small-head metal-on-metal bearings? Clin Orthop Relat Res. 2018;476:1231-7.

11. Lombardi AV Jr, Berend KR, Adams JB, Satterwhite KL. Adverse reactions to metal on metal are not exclusive to large heads in total hip arthroplasty. Clin Orthop Relat Res. 2016;474:432-40.

12. Peters RM, Van Steenbergen LN, Stevens M, Rijk PC, Bulstra SK, Zijlstra WP. The effect of bearing type on the outcome of total hip arthroplasty. Acta Orthop. 2018;89:163-9.

13. Parvizi J, Sedel L, Dunbar MJ. Clinical faceoff: instability after THA: the potential role of the bearing surface. Clin Orthop Relat Res. 2018;476:678-83.

14. Bertrand J, Delfosse D, Mai V, Awiszus F, Harnisch K, Lohmann $\mathrm{CH}$. Ceramic prosthesis surfaces induce an inflammatory cell response and fibrotic tissue changes. Bone Jt J. 2018;100B:882-90.

15. Bader R, Steinhauser E, Zimmermann S, Mittelmeier W, Scholz R, Busch R. Differences between the wear couples metal-onpolyethylene and ceramic-on-ceramic in the stability against dislocation of total hip replacement. J Mater Sci Mater Med. 2004;15:711-8.

16. Clarke MT, Lee PT, Villar RN. Dislocation after total hip replacement in relation to metal-on-metal bearing surfaces. J Bone $\mathrm{Jt}$ Surg Br. 2003;85:650-4.

17. Komistek RD, Dennis DA, Ochoa JA, Haas BD, Hammill C. In vivo comparison of hip separation after metal-on-metal or metalon-polyethylene total hip arthroplasty. J Bone Jt Surg Am. 2002;84:1836-41.

18. Salehi A, Tsai S, Pawar V, Sprague J, Hunter G, Varma SK. et al. Wettability analysis of orthopaedic materials using optical contact angle methods. Key Eng Mater. 2006;309-311:1199-202. https:// doi.org/10.4028/www.scientific.net/kem.309-311.1199.

19. Kosashvili Y, Backstein D, Safir O, Lakstein D, Gross AE. Dislocation and infection after revision total hip arthroplasty: comparison between the first and multiply revised total hip arthroplasty. J Arthroplast. 2011;26:1170-5.

\section{Affiliations}

\section{Francesco Castagnini $\mathbb{1}^{1} \cdot$ Barbara Bordini $^{2} \cdot$ Monica Cosentino ${ }^{2} \cdot$ Cristina Ancarani $^{2} \cdot$ Federica Mariotti $^{1} \cdot$ Federico Biondi ${ }^{1}$. Cesare Faldini ${ }^{3,4} \cdot$ Francesco Traina $^{4,5}$}

1 Ortopedia-Traumatologia e Chirurgia protesica e dei reimpianti d'anca e di ginocchio, IRCCS Istituto Ortopedico Rizzoli, Via Pupilli 1, 40136 Bologna, Italy

2 Laboratorio di Tecnologia Medica, IRCCS Istituto Ortopedico Rizzoli, Via di Barbiano 1/10, 40136 Bologna, Italy

3 Head of Clinica Ortopedica e Traumatologica I, IRCCS Istituto Ortopedico Rizzoli, Via Pupilli 1, 40136 Bologna, Italy
4 Orthopaedics and Traumatology, University of Bologna DIBINEM, 40123 Bologna, Italy

5 Head of Ortopedia-Traumatologia e Chirurgia protesica e dei reimpianti d'anca e di ginocchio, IRCCS Istituto Ortopedico Rizzoli, Via Pupilli 1, 40136 Bologna, Italy 